Check for updates

Cite this: RSC Adv., 2019, 9, 5362

Received 23rd November 2018

Accepted 1st February 2019

DOI: 10.1039/c8ra09652j

rsc.li/rsc-advances

\section{Cisplatin, glutathione and the third wheel: a copper-(1,10-phenanthroline) complex modulates cisplatin-GSH interactions from antagonism to synergism in cancer cells resistant to cisplatin $\dagger$}

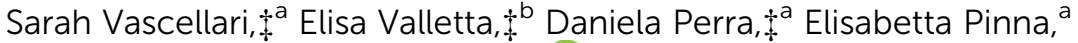
Alessandra Serra, ${ }^{a}$ Francesco Isaia, (D) ${ }^{b}$ Alessandra Pani ${ }^{\star a}$ and Tiziana Pivetta (DD *b

The antagonistic effect of glutathione (GSH) against the cytotoxicity of cisplatin was observed in both wild type and cisplatin-resistant human leukaemia and ovarian carcinoma cell lines. The simultaneous presence of the cytotoxic copper complex $\left[\mathrm{Cu}(\text { phen })_{2}\left(\mathrm{OH}_{2}\right)\right]\left(\mathrm{ClO}_{4}\right)_{2}(\mathrm{CO})$ restored the sensitivity of the cells to cisplatin, and, at selected concentrations, led to strong synergistic effects. The $\mathrm{CO}$-cisplatin-glutathione system showed a synergistic toxic effect even in the presence of $1000 \mu \mathrm{M} \mathrm{GSH}$. The three-drug cocktail exerted a higher potency against leukemic cells than against freshly isolated lymphocytes from healthy donors. Compared to actively proliferating normal lymphocytes, leukaemia cells were much more susceptible to the cytocide effect of the three-drug combination and underwent the dying process(es) much faster. When the ovarian carcinoma cells were treated with cisplatin, alone or in combination with C0, late apoptotic effects were mainly observed, suggesting that DNA interactions with the C0-cisplatin complex trigger a process of programmed cell death. In contrast, the ternary combination induced apoptotic effects similar to that shown by $\mathrm{CO}$ in single treatment, that is, early apoptosis. One possible explanation is that $\mathrm{CO}$ and cisplatin compete for GSH-binding in the culture medium. GSH in combination with $\mathrm{CO}$ and cisplatin caused a significant induction of the apoptotic process(es), through a pathway which does not compromise the integrity of the plasma membrane of cells.
\end{abstract}

\section{Introduction}

Cisplatin, $\left[\mathrm{Pt}\left(\mathrm{NH}_{3}\right)_{2} \mathrm{Cl}_{2}\right](\mathrm{CDDP})$, is a potent chemotherapeutic agent used for several types of cancer, e.g. carcinoma of the ovary, testicle, head and neck, bones, brain, lymph node, and skin. ${ }^{1,2}$ CDDP still represents the elective cytotoxic agent for the treatment of the carcinoma of the ovary, which is the leading cause of death among gynaecological cancers worldwide, with 125000 deaths annually. The high mortality rate of this type of cancer is due either to the lack of early diagnosis or to the limited number of effective chemotherapeutic agents, for which CDDP, alone or in combination with Taxol, ${ }^{3}$ often represents the only choice for clinicians. Although CDDP can also be used to treat haematological malignancies, its use is limited to

\footnotetext{
${ }^{a}$ Dipartimento di Scienze Biomediche, Università degli Studi di Cagliari, 09042 Monserrato, CA, Italy. E-mail: pania@unica.it

${ }^{b}$ Dipartimento di Scienze Chimiche e Geologiche, Università degli Studi di Cagliari, 09042 Monserrato, CA, ITALY. E-mail: tpivetta@unica.it

$\dagger$ Electronic supplementary information (ESI) available. See DOI: $10.1039 / \mathrm{c} 8 \mathrm{ra} 09652 \mathrm{j}$

\$ Equal contribution.
}

relapses by tumour cells resistant to first line drugs. CDDP is a multi-target drug $^{4-6}$ but one of the main mechanisms of action is linked to the formation of adducts with DNA. Once in the bloodstream, CDDP binds different plasmatic thiol-containing proteins ${ }^{7,8}$ however, only free CDDP molecules can enter the cells and, as the $\left[\mathrm{Pt}\left(\mathrm{NH}_{3}\right)_{2}\left(\mathrm{H}_{2} \mathrm{O}\right) \mathrm{Cl}\right]^{+}$species, react with a DNA base, ${ }^{9,10}$ thus leading to DNA distortions that activate DNA repair pathways or trigger the apoptotic cascade. ${ }^{11-14}$ The effectiveness of CDDP, as a single drug as well as in multidrug protocols, is limited by its high toxicity and by the selection, during cycles of chemotherapy, of drug-resistant cancer cell populations. The mechanisms proposed for CDDP-resistance include: (i) over-expression of drug-efflux pumps; ${ }^{15}$ (ii) increased ability to repair and/or to tolerate damaged DNA; ${ }^{16}$ (iii) overexpression of enzymes, e.g. hexokinase (HK), ${ }^{17}$ and of the STAT3/AKT pathway which promotes the Warburg effect and blocks the energetic metabolism of cancer cells via glycolysis; ${ }^{5}$ and (iv) increased intracellular levels of thiol-containing molecules like glutathione (GSH). ${ }^{11,13}$ With respect to the latter mechanism, CDDP-GSH adducts are thought to be responsible for the CDDP-resistance, ${ }^{18}$ even if the interaction of CDDP with GSH is not considered the most important 
molecular reaction of the drug within the cell. ${ }^{19} \mathrm{GSH}$ content in disease-free tissues and in cancer cells varies from micromolar to millimolar ${ }^{20,21}$ concentrations, with marked differences for the organ in question. ${ }^{22} \mathrm{GSH}$ was reported to be upregulated during the development of drug resistance, ${ }^{\mathbf{1 , 2 3}}$ however, given that higher GSH levels were not found to correlate with lower Pt(II)-DNA adducts, it has been proposed that GSH might play a role in apoptotic regulatory pathways. ${ }^{12,13,24}$

In previous works, ${ }^{25-28}$ in a wide panel of human-derived haematologic and solid tumour cell lines, we described a novel class of copper(II) complexes with 1,10-phenanthroline (phen) endowed with cytotoxic effects comparable to, and in some cases higher than, CDDP. We also reported a strong synergistic cytotoxic effect of the complex $\left[\mathrm{Cu}(\mathrm{phen})_{2}\left(\mathrm{H}_{2}\right.\right.$ $\mathrm{O})]\left(\mathrm{ClO}_{4}\right)_{2}$ (Co) in dual-drug combinations with CDDP against human T-lymphoblastic leukaemia (CCRF-CEM) cells. $^{25-27}$ These findings, coupled with data in the literature that reported an increased cellular GSH level as one of the mechanisms of CDDP-resistance, prompted us to widen our studies by investigating the effect of GSH on the cytotoxicity of $\mathbf{C o}$ in dual drug combinations, as well as the effect of ternary mixtures of $\mathrm{GSH}$, $\mathbf{C 0}$, and CDDP in order to establish whether the presence of $\mathbf{C 0}$ could influence the antagonism of GSH on CDDP. Given the potential relevance of these studies for cisplatin-based anticancer therapies, especially in CDDP-resistant cancers, the drug cocktails were tested not only against cisplatin-responsive CCRF-CEM and ovarian carcinoma (A2780) cell lines, but also against CDDP-resistant subpopulations of both CCRF-CEM (CCRF-CEM-res) and A2780 (A2780-res) cells. The main aims of the work were: (i) to strengthen the role of GSH in cisplatinresistance; and (ii) to identify combinations of CDDP, Co, and GSH with synergistic effects. Positive results would, in fact, enable the reduction of active doses of CDDP, as well as the emergence of drug-resistant cell mutants thus possibly offering an effective option to treat CDDP-resistant cancer. The concentrations of the three compounds were chosen on the basis of an experimental design (ED), whereas to study the effects of drug cocktails, and to correlate the drug concentrations with their cytotoxic activity, a model-free approach ${ }^{27}$ for data exploration and analysis, based on artificial neural networks (ANN), was used with the intent of predicting, and then experimentally confirming, the optimal drug combination(s). Finally, to determine the potential selectivity of the drug combinations toward cancer cells, the cytotoxic effects of the ternary mixtures were also tested in ex vivo cultures of human peripheral blood lymphocytes (PBLs) freshly isolated from healthy donors and compared to those in leukaemia CCRF-CEM cells.

\section{Results and discussion}

\section{Selection of cisplatin-resistant T-leukaemia CCRF-CEM cells}

A CCRF-CEM subline with a stable phenotype of CDDPresistance (CCRF-CEM-res) was obtained in about 7 months of sub-cultivation of CCRF-CEM cells in the presence of increasing concentrations of CDDP. To avoid total cell death, cells that had grown at an increased dose had to be maintained at that same concentration for 3 to 5 passages before the CDDP dose was next raised. Moreover, given that cell resistance to CDDP appears to be multifactorial (reduced drug accumulation, increased drug inactivation, enhanced DNA-repair and increased DNA-damage tolerance), ${ }^{\mathbf{1 6}}$ we deemed appropriate to obtain various mechanistically homogenous CDDP-resistant cell clones in order to study them separately. However, despite several efforts to grow cell clones by the single-cell dilution method, an otherwise successful procedure to obtain cloned cell populations resistant to cytotoxic drugs, ${ }^{29}$ single-cell cultures were not able to survive and multiply, even at CDDP doses close to its $\mathrm{CC}_{50}$. Throughout the selection time, the sensitivity of cells to CDDP was checked by the MTT method and compared to that of parental CCRFCEM (CCRF-CEMwt) cells (ESI, Table S1†). After 52 passages in the presence of CDDP it was possible to obtain a stable cell population resistant to $5 \mu \mathrm{M}$ CDDP (CCRF-CEM-res) with a Resistance Index (RI) of about 6. It is worth mentioning that cell populations growing in the presence of CDDP, as well as the final CDDP-res subline, never showed cross-resistance with the $\mathrm{Cu}$ (II) complex C0, thus suggesting different mechanism(s) of cytotoxicity and resistance by the two drugs. On the other hand, no modifications of the cell susceptibility to the reference compound Doxorubicin was ever observed.

\section{Glutathione cell content}

Given that our goal was to investigate the effect of exogenously added GSH on the cytotoxic activity of CDDP and $\mathbf{C 0}$, alone and in combination with one another, it was of vital importance to determine the basal level of GSH in all different cell lines used. Basal endogenous GSH levels were significantly lower in the CCRF-CEM cells (approximately one third) than in the A2780 cells (Fig. 1A and B). The CDDP-resistant counterpart of both cell lines showed higher contents of GSH with respect to parental cells (Fig. 1A and B). It is worth mentioning that in all our model cell lines the GSH was present almost totally in the reduced form (between 97\% and 99\%, not shown). It should also be mentioned that the GSH content values found by us are in good agreement with those reported by other authors for the same cell lines (CCRF-CEM ${ }^{23}$ and A2780 ${ }^{30,31}$ ).

\section{Artificial neural network and experimental design}

An artificial neural network (ANN) is a mathematical tool that emulates the structure of the brain and its learning ability ${ }^{27}$ (Fig. S1†). ANNs can be exploited for classification or prediction studies (i.e., this study). The results of an ANN are strictly related to the quality of the experimental data and, in this context, the experimental design ${ }^{32}$ determines the choice of the minimum set of data with the highest content of information. The concentrations of the drugs were chosen to be in the range of 0 to twice the $\mathrm{CC}_{50}$, preliminarily determined, following the experimental design reported in the ESI (Fig. S2). $\uparrow \mathbf{C 0}, \mathrm{GSH}$ and CDDP were respectively evaluated on wild type cells in the ranges $0-2,0-1000$, and $0-2 \mu \mathrm{M}$, and on the CDDP-res cells in the ranges $0-2,0-1000$, and $0-15 \mu \mathrm{M}$. Drug concentrations and cytotoxicity values were used to form the data matrix; drug concentrations were used as input data and cytotoxic activity 

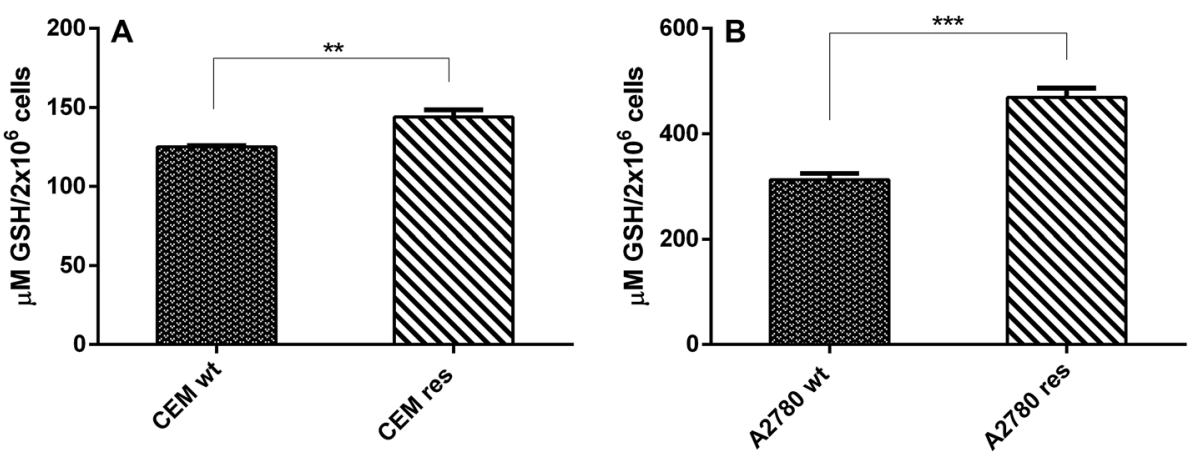

Fig. 1 GSH content in CDDP-sensitive and CDDP-resistant T-leukemia CCRF-CEM (A) and A2780 (B) cells. GSH level was determined by a fluorimetric kit and data represent the mean \pm SD of three independent experiments. Values were expressed in $\mu \mathrm{m}$ of GSH/2 $\times 10^{6}$ cells. Statistical analysis was performed using $t$ test on grah pad prism $2016 ; * * p<0.001 ; * * * p<0,0001$.

values as output data. The generalization ability of the network was used to predict the cytotoxicity on the whole working space according to a bi-dimensional grid with 40 points per side for the binary systems, and according to a cube with 20 points per side for the ternary system. In this way, the cytotoxicity of 1600 (grid of $40 \times 40$ ) and 8000 (cube of $20 \times 20 \times 20$ ) virtual drug combinations were predicted. At first, the data of binary systems were processed individually, and subsequently, all the data of binary and ternary systems were processed together. The results for the binary systems obtained in the two processes were strictly comparable, proving the robustness and the reliability of the method. A three layers structure with 4 (for binary systems) or 5 (for ternary systems) neurons in the hidden layer was sufficient to model the experimental data. The training of the network was checked by comparing calculated and experimental values of the output data. The good agreement between calculated and experimental values for the tested cells is shown in Fig. S3. $\dagger$ The trained network was used to predict the cytotoxicity values of single drugs and mixtures for the whole working space. From these predicted values, the dose-response curves for each compound and the cytotoxicity surfaces for binary and ternary systems were built; finally, the synergistic/ antagonistic effect was calculated according to Pivetta et al. ${ }^{27}$ In brief, since the additive effect of two or more drugs is not the algebraic summation of their cytotoxic activities, the NonAlgebraic Additive Effect (NAEE) was considered, that is an index that represents an operational definition of the additive effect, calculated according to a sequential action of the drugs. Synergy or antagonism was then determined by calculating the Net Multi-Drug Effect Index (NMDEI); i.e. the difference between the predicted cytotoxic activity and the NAEE, calculated for all the points of the grids, for binary and ternary systems. The NMDEI values allow a surface that shows the possible interactions occurring among drugs to be defined; i.e. synergistic (positive values), additive (zero values), or antagonistic (negative values) effects.

\section{Cytotoxic effects of CDDP, C0 and GSH}

In Fig. 2, the calculated dose/response curves for CDDP, GSH and $\mathbf{C O}$ are shown. As can be seen, there is good agreement between calculated (line) and experimental points $(\bullet)$. The different shapes of the curves suggest different modes of action exerted by the drugs. The calculated drug concentrations required to inhibit the cell proliferation by $50 \%\left(\mathrm{CC}_{50}\right)$ are reported in Table 1.

As can be seen in Fig. 2 and Table 1, CDDP was active against both wild type leukaemia CCRF-CEM and ovarian carcinoma A2780 cell lines with a comparable potency, while it was less active against their CDDP-resistant cell counterparts. C0 was active against all cell lines tested, but showed the highest activity against A2780 cells; GSH displayed a moderate activity against CCRF-CEM cells. It should not be surprising that GSH showed some cytotoxicity; in fact, although most studies showed that GSH is an anti-apoptotic agent, it was also reported to exert cytotoxic activities towards cancer cell lines by inducing DNA fragmentation ${ }^{33}$ or by interfering with the cellular progression stage. ${ }^{34}$ As observed by Balendiran, ${ }^{35}$ with respect to cancer, GSH metabolism is able to play both protective and pathogenic roles and, as a matter of fact, different chemotherapy responses in normal versus tumour cells were obtained, providing a possible GSH-based strategy for the protection of normal cells. ${ }^{36}$ In summary, the role of GSH in cancer is complex and its behaviour depends on several factors, including the polymorphisms of the type of cancer and the individual GSH homeostatic regulation and needs of each patient.

\section{Binary mixtures}

The plot of cytotoxicity (\% values) as a function of the drug concentrations for the 1600 virtual combinations defines the response surface. For the wild type and CDDP-resistant CCRFCEM cells, the response surfaces are shown in Fig. 3 and the results relative to the A2780 cell lines are reported in the ESI (Fig. S4). $\dagger$ The experimental data (grey cubes) are superimposed on the calculated surfaces to show whether there is agreement between estimated and measured values.

As can be seen in Fig. 3 and $\mathbf{S} 4, \dagger$ the cytotoxic effect increases with the concentrations of the two drugs, with a trend resembling that of a dose-response curve. The analysis of the surfaces indicates that: (i) the mixture C0-CDDP acts in a similar way on 

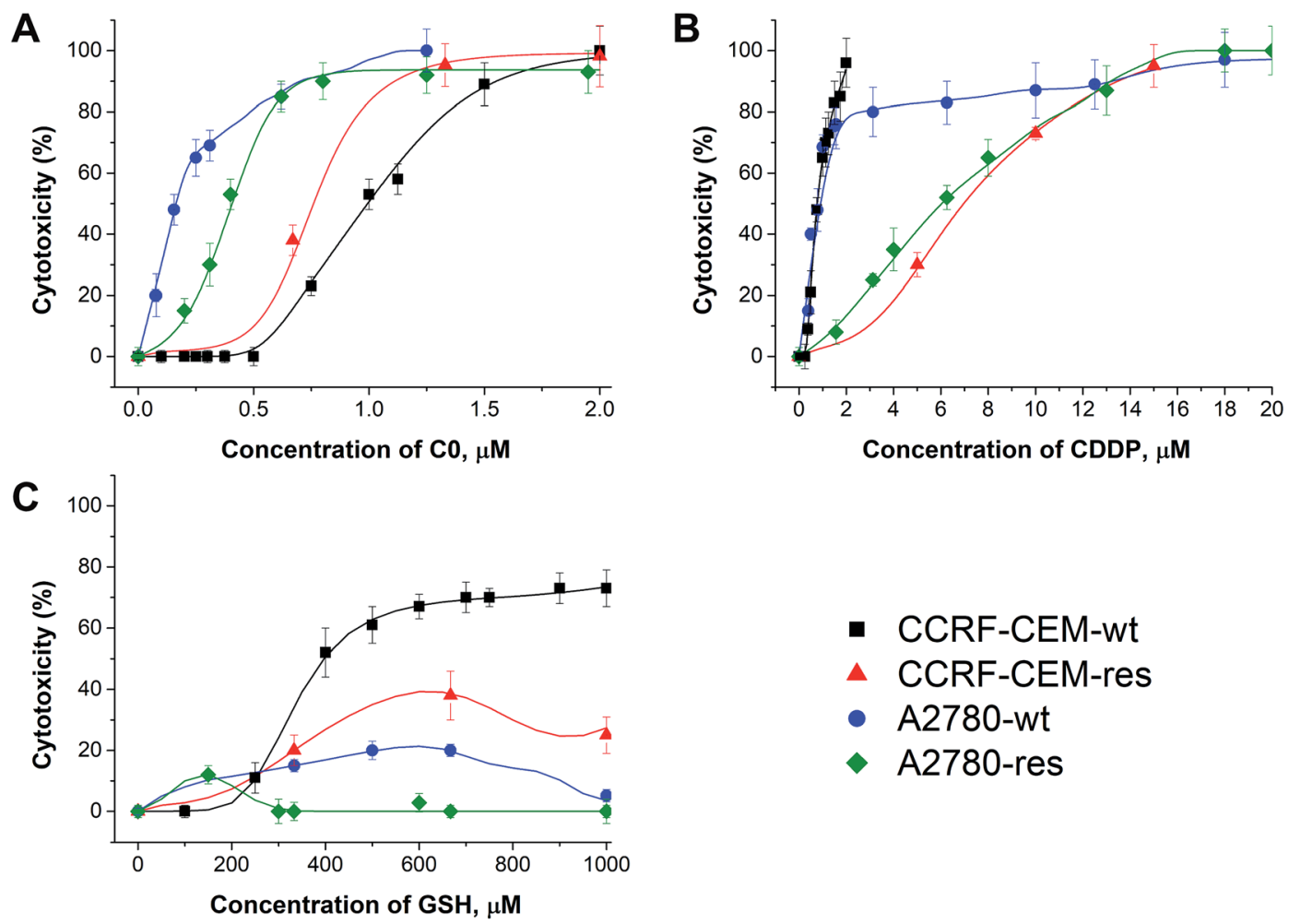

Fig. 2 Dose-response curves of CO (A), CDDP (B), and GSH (C) calculated by the network (line) and experimental points against wild type and cisplatin-resistant CCRF-CEM and A2780 cell lines.

Table 1 Concentrations of single drugs of CO, CDDP and GSH required to inhibit the cell proliferation by $50 \%\left(C_{50}, \mu \mathrm{M}\right)$ towards wild type and cisplatin-resistant CCRF-CEM and A2780 cells

$\mathrm{CC}_{50}, \mu \mathrm{M}$

\begin{tabular}{lllll} 
Compound & CCRF-CEM-wt & CCRF-CEM-res & A2780-wt & A2780-res \\
\hline Co & $0.97 \pm 0.02$ & $0.76 \pm 0.01$ & $0.15 \pm 0.01$ & $0.37 \pm 0.03$ \\
CDDP & $0.71 \pm 0.02$ & $6.51 \pm 0.05$ & $0.90 \pm 0.02$ & $5.90 \pm 0.05$ \\
GSH & $\geq 400$ & $\geq 400$ & n.d. & n.d.
\end{tabular}

wild type and cisplatin resistant cells (Fig. 3A, D, S4A and S4D †); (ii) when CDDP is in the presence of GSH, its cytotoxicity decreases, and higher doses are required to reach the same extent of mortality; this is particularly evident in the CDDPresistant cells in which to obtain $\approx 50 \%$ toxicity doses of $\mathrm{CDDP} \geq 10 \mu \mathrm{M}$ are required, compared to a $\mathrm{CC}_{50}$ of $\mathrm{CDDP}$ alone of $6.51 \mu \mathrm{M}$ (Fig. 3B, E, S4B and S4E); and, by contrast (iii) the presence of GSH increases the cytotoxicity of $\mathbf{C o}$. In the contour plots, reported on the bottom planes of the surfaces, the areas of cytotoxic iso-values can be explored to choose the combinations with the desired level of cytotoxicity to identify the corresponding dose of each drug. Selected examples of binary combinations that exert a 50\% cytotoxicity are reported in Table 2.

To better understand if the drugs act synergistically, the surfaces calculated by the NMDEI for the binary combinations of C0-CDDP, C0-GSH and CDDP-GSH in the CCRF-CEM cells, are reported in Fig. 4. The results for the A2780 cells are reported in the ESI (Fig. S5). $\dagger$

Analysing the surfaces reported in Fig. 4, and recalling that NMDEI assumes positive values for synergistic effects, zero values for additive effects and negative values for antagonistic effects, it is possible to observe that:

\section{In CCRF-CEM cells}

$C D D P-G S H$. The plot of NMDEI as a function of CDDP and GSH concentrations (Fig. 4A) reveals a wide area of negative values in the range $\mathrm{CDDP} \geq 0.76 \mu \mathrm{M}$ and $\mathrm{GSH} \geq 384 \mu \mathrm{M}$, indicating antagonistic CDDP-GSH interactions. This is more relevant in the CDDP-resistant cells (Fig. 4D), and particularly at high GSH concentrations. In fact, a combination of $15 \mu \mathrm{M}$ CDDP and $950 \mu \mathrm{M}$ GSH shows a $42 \%$ mortality (NMDEI is -53.34), whereas at the same concentrations in single drug treatments, the two drugs induce cell death by up to $94.4 \%$ and $24.9 \%$, respectively (Fig. 3E).

CDDP-CO. The synergistic effect of $\mathbf{C O}$ in combination with CDDP (Fig. 4B) in the wild type cell population is evidenced by a wide positive area, with a maximum NMDEI of $91.9 \%$ at 0.34 $\mu \mathrm{M}$ CDDP and $0.54 \mu \mathrm{M}$ Co. This combination shows a cytotoxicity of $96.7 \%$, while the two compounds used alone at the same concentrations of the combination, showed mortality values of only $3 \%$ and $6 \%$, respectively. No negative values were observed. The synergism between the two drugs is revealed also in the CDDP-resistant counterpart (Fig. $4 \mathrm{E}$ ) by a wide positive area with a peak at $0.74 \mu \mathrm{M} \mathrm{CO}$ and $4.74 \mu \mathrm{M}$ CDDP (NMDEI is $+28 \%$ ). This combination shows mortality of $87.8 \%$, while the two 

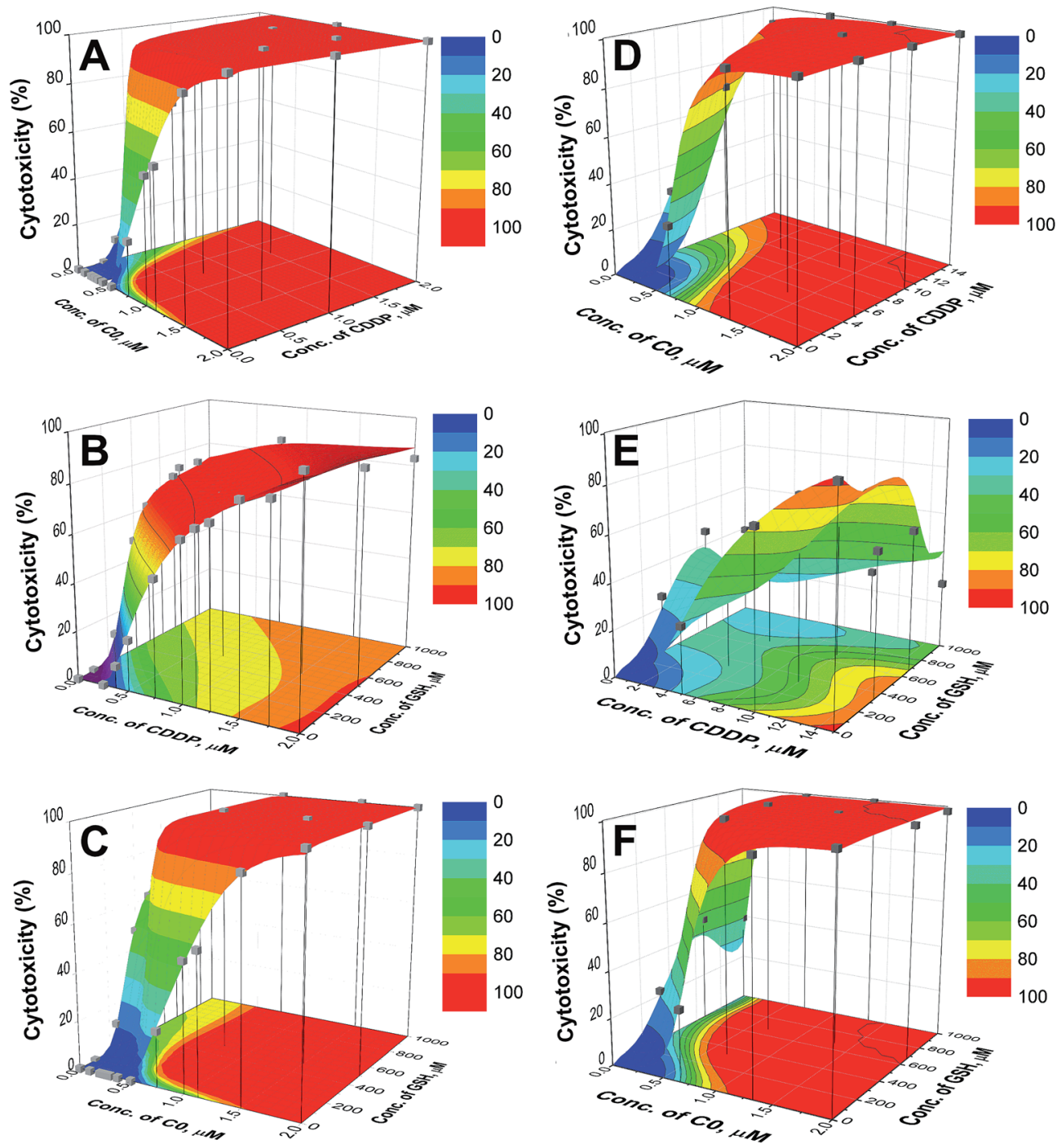

Fig. 3 Response surfaces for C0-CDDP (A and D), CDDP-GSH (B and E) and C0-GSH (C and F) for CCRF-CEM-wt (left) and CCRF-CEM-res (right) cell lines. The experimental points $(\boldsymbol{\square})$ are superimposed to the surfaces. The contour plots of cytotoxicity iso-values are shown in the bottom planes.

drugs used alone show cytotoxicity values of $45.5 \%$ and $25.7 \%$, respectively (Fig. 3D).

Co-GSH. Co revealed synergistic activity (Fig. 4C) also in combination with GSH towards wild type cells, with a maximum (NMDEI $+82 \%$; mortality $98 \%$ ) at $0.60 \mu \mathrm{M} \mathrm{CO}$ and $220 \mu \mathrm{M} \mathrm{GSH}$. At these concentrations, the two drugs, used alone, showed mortality values of $8 \%$ and $6 \%$ respectively. The synergistic effect is exerted also on resistant cells (Fig. $4 \mathrm{~F}$ ) with two maxima of activity: the first at $0.42 \mu \mathrm{M} \mathrm{CO}$ and $900 \mu \mathrm{M}$ GSH (NMDEI $+70.9 \%$ ) and the second at $0.53 \mu \mathrm{M} \mathrm{CO}$ and $350 \mu \mathrm{M} \mathrm{GSH}$ (NMDEI $+64.9 \%)$. This latter drug combination exhibits a cytotoxicity value of $95.6 \%$, while the two drugs used alone at the same concentrations showed a cytotoxicity of only $10.8 \%$ and $21.8 \%$, respectively (Fig. 3F).

Table 2 Selected combinations of two drugs that inhibit the cell proliferation by $50 \%\left(C_{50}, \mu M\right)$ towards wild type and cisplatin-resistant CCRFCEM and A2780 cells

\begin{tabular}{|c|c|c|c|c|}
\hline \multicolumn{5}{|l|}{$\mathrm{CC}_{50}, \mu \mathrm{M}$} \\
\hline $\mathrm{CDDP}+\mathrm{GSH}$ & $0.32+200$ & $10+300$ & $7.0+400$ & $5.3+50 ; 12+350 ; 21+900$ \\
\hline $\mathrm{Co}+\mathrm{GSH}$ & $0.42+200$ & $0.35+300$ & $0.10+300$ & $0.26+200$ \\
\hline
\end{tabular}




\section{CCRF-CEM-wt}
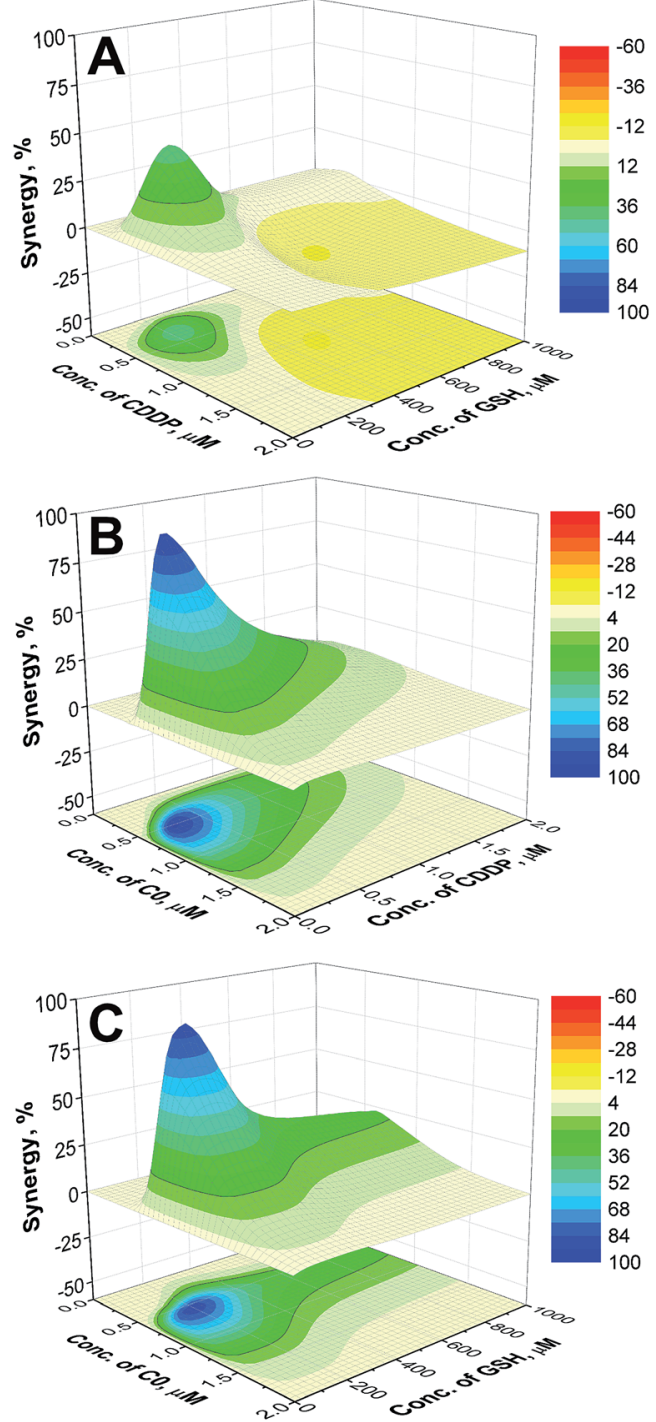

CCRF-CEM-res
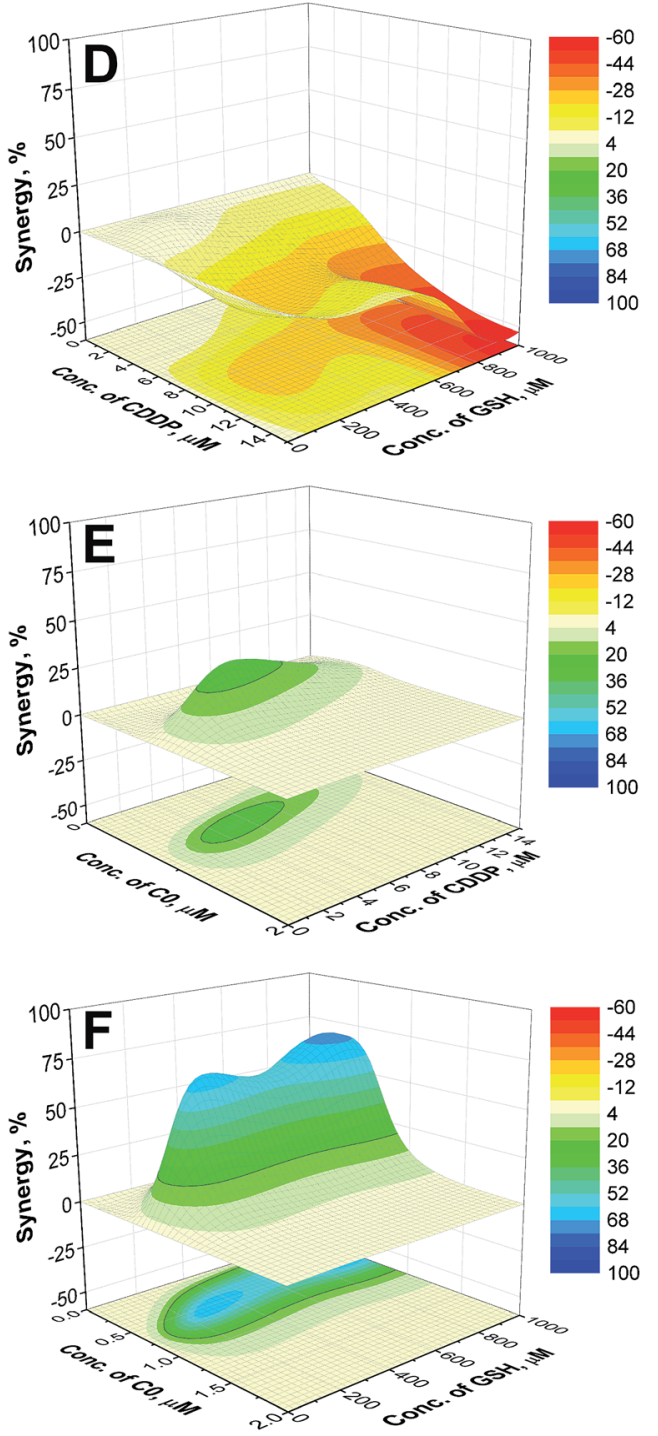

Fig. 4 Synergistic surfaces for the binary systems CDDP-GSH (A and D), C0-CDDP (B and E) and C0-GSH (C and F) in CCRF-CEM-wt (left) and CCRF-CEM-res (right) cell lines (red, white and blue colors indicate antagonistic, additive and synergistic drug interactions, respectively).

In A2780 cells. The association of CDDP and GSH confirmed antagonistic drug interactions also against the ovarian carcinoma A2780 cells, and in particular, against the CDDP-resistant subline (NMDEI $-26 \%$ for wild type and $<0$ for all the combinations for resistant cells, Fig. S5B and S5E $\dagger$ ). By contrast, in these cell lines the presence of GSH in Co-treated cells (Fig. S5C and $\mathrm{S} 5 \mathrm{~F} \dagger$ ) resulted in strong synergistic cytotoxic effects (NMDEI $+54 \%$ for GSH $1000 \mu \mathrm{M}$ and Co $0.13 \mu \mathrm{M}$, and $+18 \%$ for GSH $550 \mu \mathrm{M}$ and C0 $0.33 \mu \mathrm{M}$ ), and again particularly in the A2780-res subline (NMDEI $+86 \%$ for CO $0.26 \mu \mathrm{M}$ and GSH 550 $\mu \mathrm{M})$. In combination with CDDP, CO showed additive effects (NMDEI is $\approx 6 \%$ ) in the A2780-wt, and low synergistic effects in the A2780-res cells (NMDEI $+12 \%$ for Co $0.26 \mu \mathrm{M}$ and CDDP 10.5 $\mu \mathrm{M}$; see Fig. S5A and S5D $\dagger$ ).

\section{Ternary mixtures}

For the evaluation of the three-drug system, a cubic surface was built up. The $X, Y$ and $Z$ axes indicate the concentrations of the three different drugs, while NMDEIs were represented by red, white, and blue coloured points indicating antagonistic, additive, and synergistic effects, respectively. In the reported surfaces (Fig. 5), the concentration of $\mathbf{C 0}$ varies along the $Z$ axis giving visual evidence of its influence on the cytotoxicity of CDDP-GSH mixtures both against wild type and CDDP-resistant CCRF-CEM and A2780 cell lines. To facilitate the readability of the results, only 6 planes, representing six different concentrations of $\mathbf{C 0}$, are presented.

As can be seen in Fig. 5, in the absence of C0 (bottom plane), the antagonistic effect of GSH on CDDP was the rule, especially at high GSH doses (yellow and red cubes). However, when cells were exposed to CDDP and GSH in the presence of 


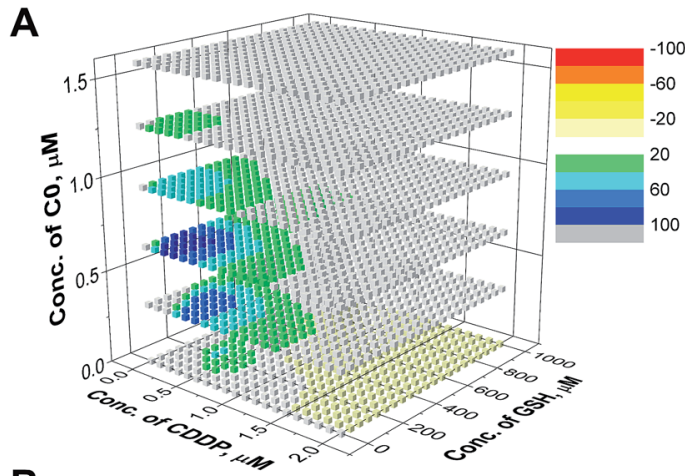

B

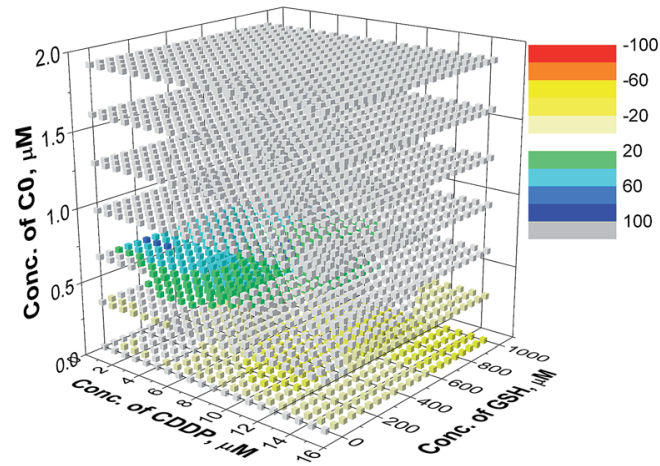

C

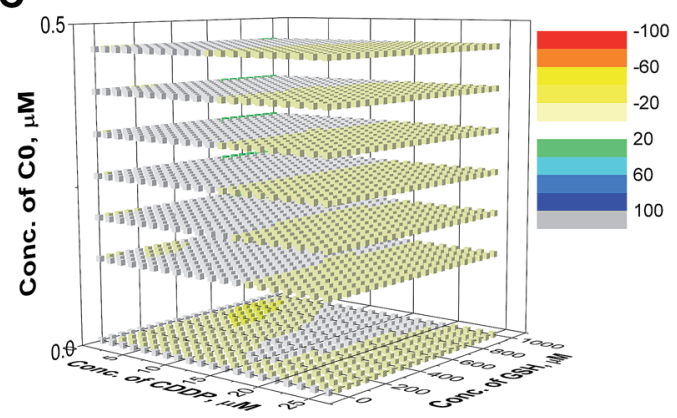

D

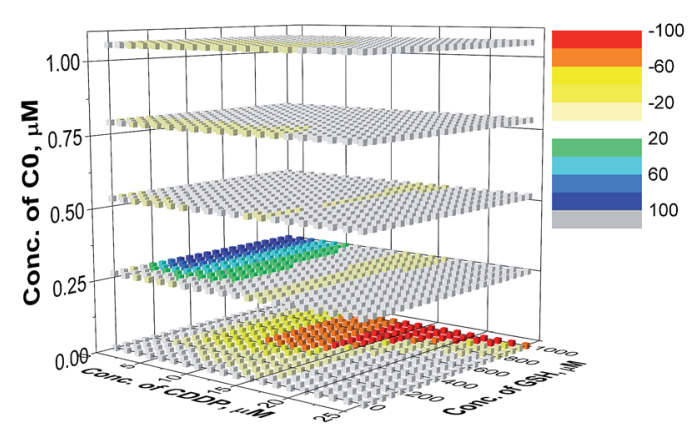

Fig. 5 Synergistic surfaces for the ternary system CDDP-GSH-C0 in (A) CCRF-CEM-wt, (B) CCRF-CEM-res, (C) A2780-wt and (D) A2780-res cell line. Red, white, and blue colors indicate antagonistic, additive, and synergistic effects, respectively.

concentrations of $\mathbf{C O}$ higher than $\approx 0.25 \mu \mathrm{M}$, the antagonism exerted by GSH on the CDDP activity disappeared (white cubes) and, at a number of specific dose-combinations, synergistic effects were revealed (green and blue cubes).

To better evidence the reverting effect of $\mathbf{C O}$ against the antagonism of GSH on CDDP, the cytotoxic activity of (i) CDDP alone, (ii) CDDP in binary combination with $300 \mu \mathrm{M}$ GSH, and (iii) CDDP in ternary combination with $300 \mu \mathrm{M} \mathrm{GSH}$ and 0.30 or $0.25 \mu \mathrm{M}$ of $\mathbf{C 0}$, is reported in Fig. 6 for both resistant cell lines. As can be seen, in the presence of GSH alone, the potency of CDDP decreased (compare the black and red lines), but if $\mathbf{C 0}$ was present, the full cytotoxic CDDP potential was restored, or increased (blue lines), particularly at low CDDP concentrations. Nevertheless, it has to be noted that it is not appropriate to consider the most synergistic combination as the optimal one, since the choice may depend on several factors, such as the individual tolerated concentration of CDDP and/or the individual concentration of endogenous GSH.

\section{Check of the ED-ANN results}

To check the reliability of the proposed approach (ED and ANN), the predicted drug-combinations giving the most relevant effects were prepared and experimentally evaluated. The biological activity predicted by the network was confirmed, and the
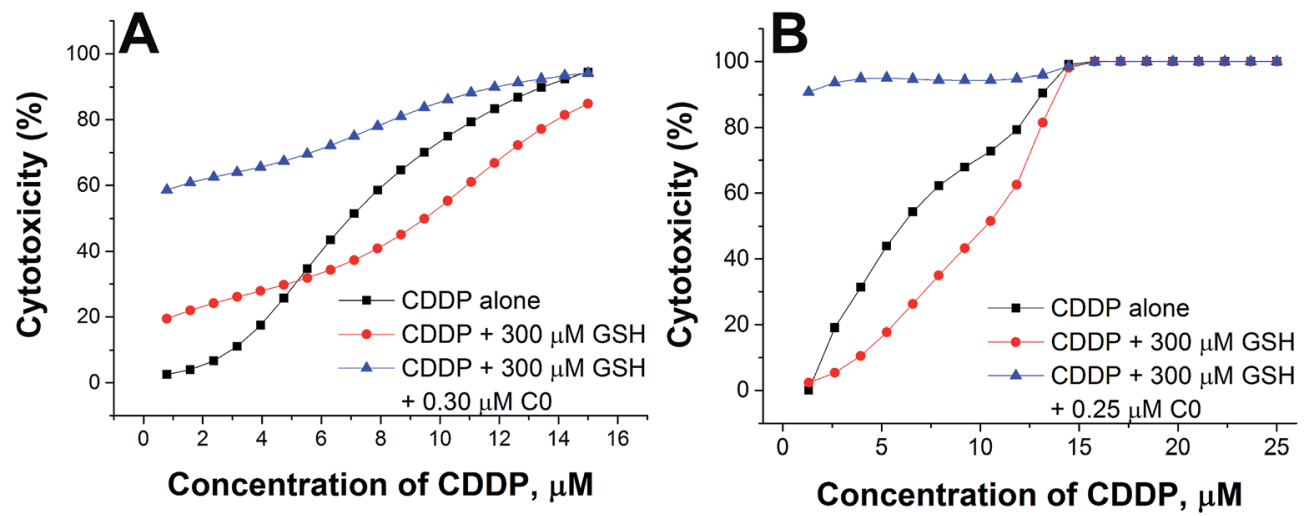

Fig. 6 Cytotoxic activity of CDDP alone, in binary combination with GSH, or in ternary combination with GSH and CO in CCRF-CEM-res (A) and A2780-res (B) cells. 
most powerful combinations were used for the successive experiments.

\section{Cytotoxicity of drug cocktails in ex vivo normal human PBLs}

The strong synergism shown by CDDP-Co-GSH combinations against the T-leukaemia CCRF-CEM cells, which also holds in the CDDP-resistant subline (CCRF-CEM-res), prompted us to determine the effect of the ternary drug combination against ex vivo normal peripheral lymphocytes, the natural control cells for leukaemia cells, so as to have a faithful estimate of the selectivity of action of these drug-cocktails. To this end, human peripheral blood lymphocytes (PBLs) freshly isolated from blood samples of healthy donors were seeded as such (i.e. resting PBLs), in the presence of the mitogen PHA (i.e. PHAactivated PBLs), or in the presence of both PHA and Interleukin-2 (i.e. PHA/IL2-stimulated PBLs). Each culture was then incubated in the presence of the most synergistic threedrug combination against CCRF-CEM-wt cells; i.e. $0.5 \mu \mathrm{M}$ CDDP, $0.5 \mu \mathrm{M}$ Co, and $300 \mu \mathrm{M}$ GSH. Duplicate PBL cultures were incubated in the presence of each drug alone at the same concentration used in the combination. For comparative purposes, cultures of CCRF-CEM were run under the same experimental conditions. The number of viable cells was determined after 24,48 and $72 \mathrm{~h}$ of incubation by the trypan blue exclusion method. Data were reported as percentages of untreated controls (Fig. 7). The PBL values in Fig. 7 are the mean data of independent experiments performed with PBLs isolated from three different donors. In Fig. 8, growth curves and viable cell counts of untreated $v s$. drug treated PBLs isolated from one single donor are shown.

Single-drug treatments with $0.5 \mu \mathrm{M}$ CDDP showed a cytotoxic effect increasing over time only against CCRF-CEM cells and active/proliferating cell cultures (i.e. PHA-activated and PHA/IL2-stimulated PBLs), while, as expected, it had only negligible effects against non-activated/non-proliferating cells (i.e. resting-PBLs). After $72 \mathrm{~h}$ of incubation, the degree of cytotoxicity of CDDP was comparable in PBLs and CCRF-CEM cells, with an average of $60-70 \%$ cell viability, with respect to untreated controls. In the PBL cultures, however, CDDP led to cell death earlier (i.e. at $24 \mathrm{~h}$ in PHA/IL2-stimulated; at $48 \mathrm{~h}$ in PHA-activated) than in the CCRF-CEM cultures, with viable CCRF-CEM cells still being over $80 \%$ of controls at $24 \mathrm{~h}$, and over $70 \%$ at $48 \mathrm{~h}$. The cytotoxic effect of $0.5 \mu \mathrm{M}$ CO in PBLs seemed to better correlate with the activation stage of the cells; the viability of PHA/IL2-stimulated PBLs was reduced by $30 \%$ already after $24 \mathrm{~h}$, while that of the PHA-activated PBLs by 25$30 \%$ only after $48 \mathrm{~h}$. In contrast to CDDP, CO seemed to affect the viability also of the resting PBLs as a slight toxic effect, i.e. from $10 \%$ to $20 \%$ cytotoxic effects were observed in each PBL
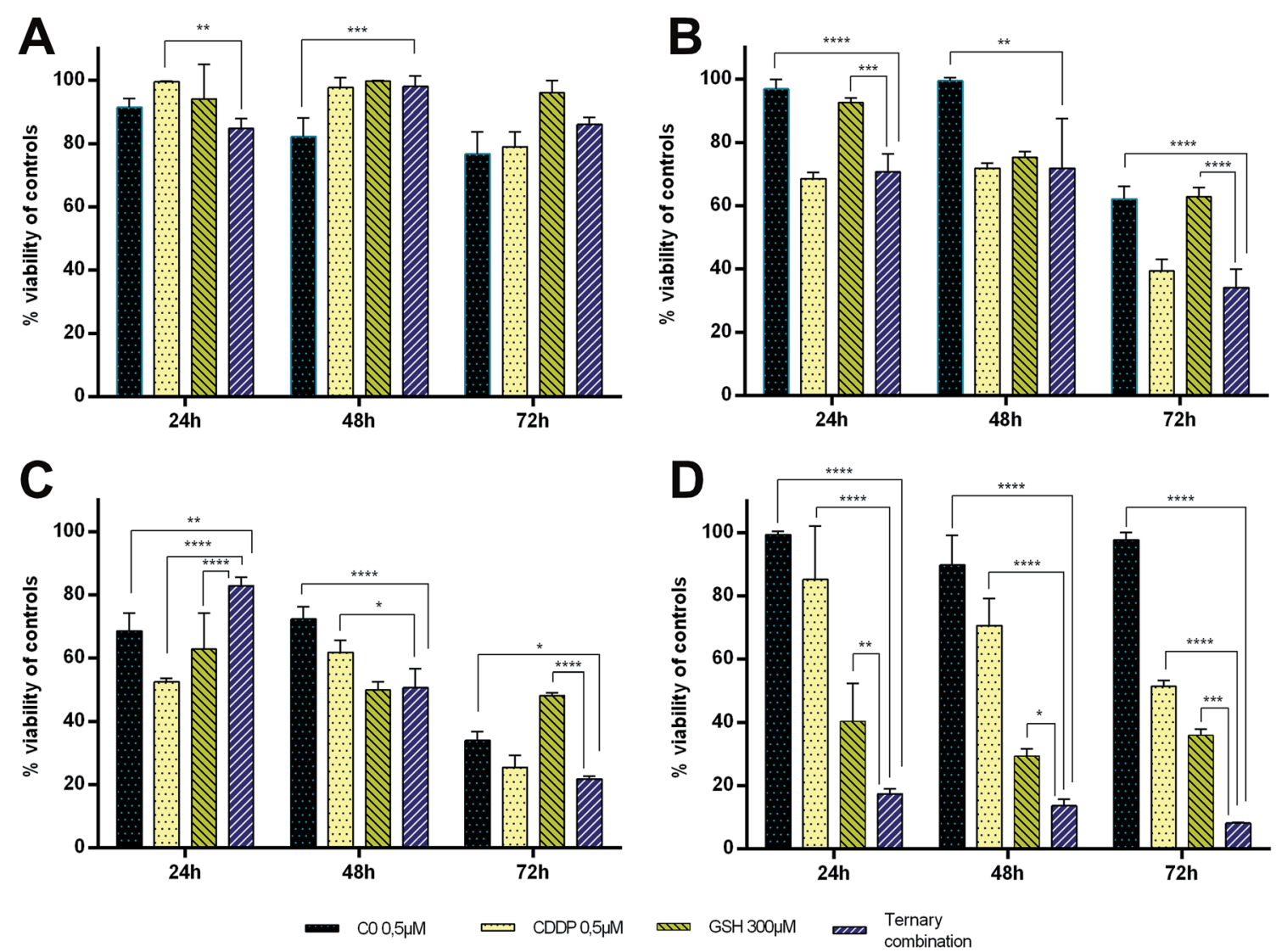

Fig. 7 Cytotoxic activity of CO, CDDP and GSH, alone and in ternary combination against PBLS and CCRF-CEM cultures after 24,48 and 72 h of drug treatment. (A) Resting PBLs, (B) PHA-stimulated PBLs, (C) PHA + IL2-stimulated PBLs, (D) CCRF-CEM-wt. Values obtained in drug-treated samples were expressed as percentages of their respective controls. 

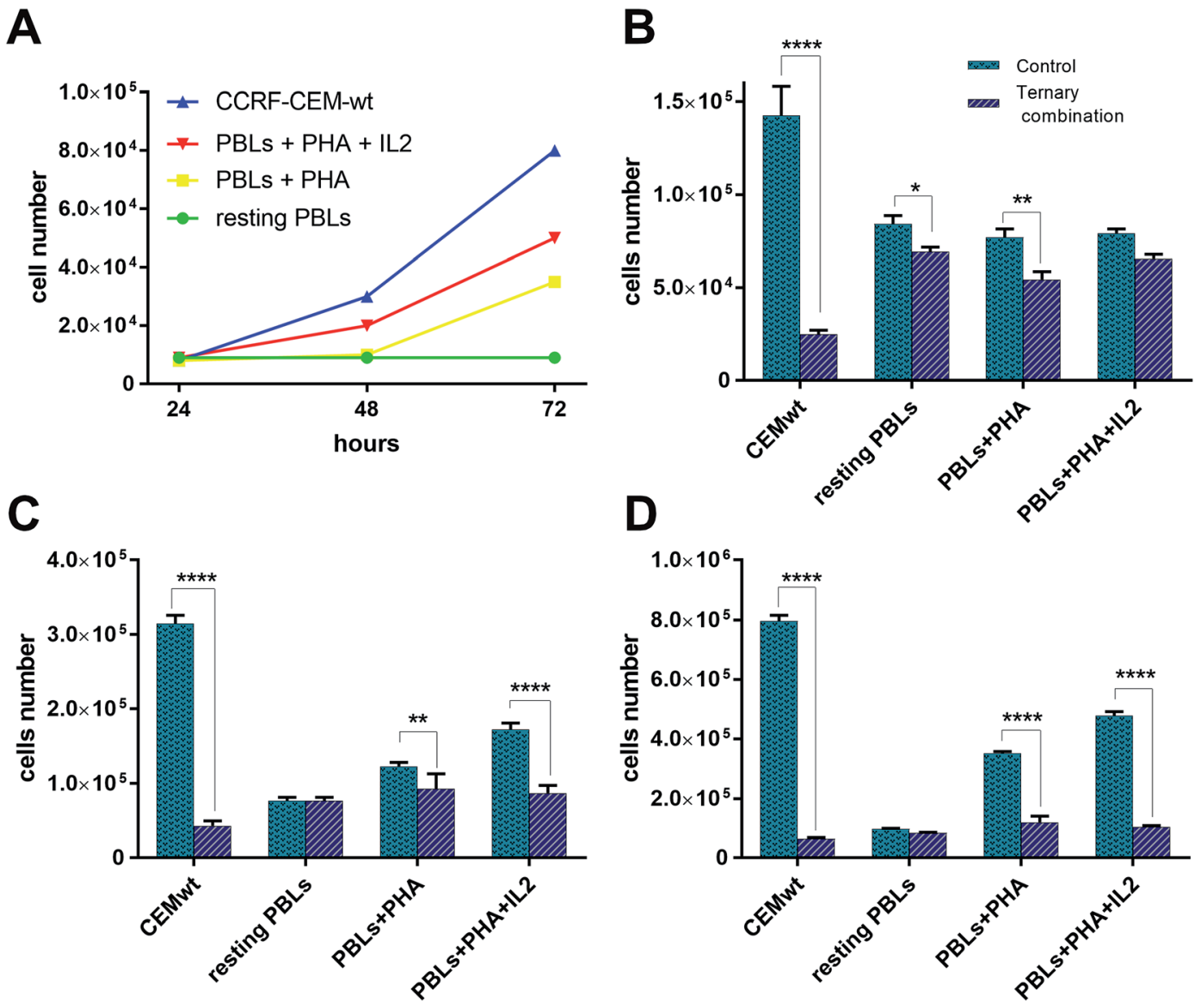

Fig. 8 Growth curves and viable cell number counts of untreated vs. combination-treated CCRF-CEM and PBL cell cultures. (A) Growth curves of CCRF-CEM-wt cells and PBLs. Numbers of viable cell in untreated vs. ternary combination-treated CCRF-CEM-wt cells and PBLs after $24 \mathrm{~h}$ (B), $48 \mathrm{~h}(\mathrm{C})$, and $72 \mathrm{~h}(\mathrm{D})$.

preparation from the three different donors. Nonetheless, in CCRF-CEM cells the cytotoxicity of $\mathbf{C O}$ appeared to be even lower, if any, than in the resting PBLs: the viability of treated CCRF-CEM cells being over $90 \%$ of controls at all time points considered.

GSH-treatments $(300 \mu \mathrm{M})$ appeared to be more toxic in CCRF-CEM cells than in PBLs and, among the latter, the PHA/ IL2-stimulated PBLs were affected the most, followed by the PHA-activated PBLs, whereas resting PBLs were not affected at all. Although the GSH cytotoxicity was slightly greater against CEM cells (60-70\% mortality) than against PHA/IL2-stimulated PBLs (50\% mortality), in both cell types the maximum effect was reached after $48 \mathrm{~h}$ of exposure to GSH. Compared to single-drug treatments, in CCRF-CEM cells the drug cocktail confirmed a strong synergistic effect with a mortality of over $80 \%$ already at $24 \mathrm{~h}$, further increasing with time. As for the PBLs, the PHA/IL2stimulated PBLs were the most sensitive of all to the toxic effect of the drug combination, that was, however, both less potent and more delayed with respect to that exerted against CCRFCEM cells; i.e. a maximum of only $50 \%$ mortality after $48 \mathrm{~h}$. The toxic effect against the PHA-activated PBLs was even slower than that shown in the PHA/IL2-stimulated PBLs; i.e. 20-30\% mortality at the first two time points, and comparable only after
$72 \mathrm{~h}$. The drug cocktail was totally devoid of cytotoxicity on the resting PBLs at any time points considered. Moreover, if we consider the effects of the drug combination $v s$. single-drug treatments in the PBLs, the drug cocktail always showed a degree of cytotoxicity comparable to that of the CDDP alone, whereas in the CCRF-CEM cells the toxic effect of the combination was 7 -fold greater than that of CDDP alone. Taken together, these findings are very promising since the ternary combination showed a clear selective cytotoxic effect for Tleukaemia cells. In addition to the higher activity against leukaemia CEM cells ( $8 \%$ residual viability after $72 \mathrm{~h}$ ) compared to PHA/IL2-stimulated PBLs (50\% viability at $72 \mathrm{~h}$ ), the effect of the drug combination against CCRF-CEM cells appeared to be also exceptional: i.e. only $17 \%$ viability left after $24 \mathrm{~h}$, compared to the $80 \%$ of the proliferating PBLs at the same time point.

\section{Multi-drug resistance (MDR) activity}

To establish whether the CDDP-resistance of CCRF-CEM res and A2780 res sublines was due to the selection of MDR cell populations, the potential overexpression of drug-efflux pumps, MDR1/P-gp and MRP1, was investigated in drug-resistant cell lines and compared to that parental by a specific MDR fluorimetric kit. However, no significant decrease ( $\leq 10 \%$ variation) in 
RFU values of the CDDP-resistant $v s$. the wild type cells was detected (Fig. 9), thus indicating that a MDR phenotype was not the major mechanism of CDDP resistance.

\section{Apoptosis induction by ternary drug combination in $\mathbf{A 2 7 8 0}$ cells}

To determine whether the strong synergistic effect of the drug combinations was due to a massive induction of apoptosis, A2780 cells were exposed to CDDP, Co and GSH, alone or in combination one another. Moreover, early and late apoptosis events were determined at different time points by the Alexa Fluor 488 Annexin V and PI staining method, which allows an excellent differentiation between early and late apoptotic cells. ${ }^{37}$ Cells were scored as early apoptotic or late apoptotic based on Annexin V-positive staining only, or both Annexin V and PI positivity, respectively. Conversely, viable cells were scored by the absence of Annexin $\mathrm{V}$ binding associated to propidium uptake.

As shown (Fig. 10), in single treatments both C0 and CDDP induced apoptosis in A2780-res cells. After 20 hours of treatment, Co-treated cells (Fig. 10B) were Annexin V-positive and Annexin V-PI-positive, indicating cells in both early and late apoptosis. As expected, CDDP, known to induce apoptosis in several normal and tumour cells via the formation of platinumDNA adducts, ${ }^{\mathbf{1 1 - 1 4}}$ induced apoptosis also in the A2780-res cells (Fig. 10C). However, at the same time point, in contrast to Cotreated cells, most apoptotic cells exposed to CDDP were in the late apoptotic phase, showing both Annexin V and PI positive staining. A few cells showed membrane damage and appeared red-stained by the intercalating dye PI. A similar pattern was obtained when cells were co-treated with CDDP and Co in a dual-drug combination (Fig. 10D); also in this case, in fact, the majority of the apoptotic cell fraction was positive for both stains, indicating late apoptosis. By contrast, at the same time point, when GSH was associated to CDDP and Co (Fig. 10E) a statistically significant fraction of apoptotic cells appeared to be blocked in the early apoptotic phase. In the three-drugtreated cultures, almost all cells showed an intact cytoplasmic membrane, as indicated by membranes positive for Annexin V associated with the absence of PI staining. After 20 hours of exposure to the ternary drug cocktail (Fig. 9F), the percentage of early apoptotic cells, significantly increased from $9 \%$ to $96 \%$ ( $p$ $<0.001$ ). Similarly, an increased percentage of early apoptotic cells was observed when the cells were treated with Co alone (from $9 \%$ to $80 \%, p<0.001$ ). By contrast, the level of apoptosis in the early phase did not significantly change after exposure to CDDP alone or CDDP-Co co-treatment, while late apoptosis levels significantly increased from $9 \%$ to $65 \%(p<0.001)$ and to $60 \%(p<0.001)$, respectively. Similar results were obtained in the parental A2780wt cells (data not shown).

As far as the mode of action of drugs with one another in the binary and ternary combinations are concerned, a number of considerations needs to be drawn. As already mentioned, CDDP is a multi-target agent that interacts with several cellular molecules, therefore, synergistic effects with $\mathbf{C 0}$ may arise from interaction of the copper-complex with other molecules and/or processes correlated with one of the different molecular targets of CDDP. Given that $\mathbf{C o}$ can also bind $\mathrm{DNA}^{28}$ by intercalation, and we previously demonstrated ${ }^{38}$ that copper-platinum complexes can readily be formed by mixing solutions of CDDP and $\mathbf{C O}$ at various drug concentrations, once inside the cell, copper-platinum-DNA adducts may readily add to those of platinum-DNA and copper-DNA. An alternative or probably an additional mechanism of the CDDP-C0 synergism may reside in the inhibitory effect of these two drugs at different levels of the regulatory pathway that in cancer cells activates and maintains glycolysis as the main energetic metabolism also in the presence of oxygen (i.e. the Warburg effect). ${ }^{39}$ Although a detailed description of this metabolic switch in cancer cells, as well as its significance, complex molecular regulation, is beyond the aim of this study, it is worth mentioning that (i) the Warburg effect is activated by an increased expression of pyruvate kinase (PDK), an enzyme that inhibits the flux of pyruvate into the Krebs' cycle; and (ii) one of the reported cellular targets of CDDP is the synthesis of PDK itself. Besides the metabolic advantages of the Warburg effect, the inhibition of oxidative phosphorylation (OXPHOS) allows cancer cells to reduce ROS generation, and their triggering effect on the p53-mediated apoptotic process. Thus, the synergistic interaction of $\mathbf{C 0}$ with CDDP may

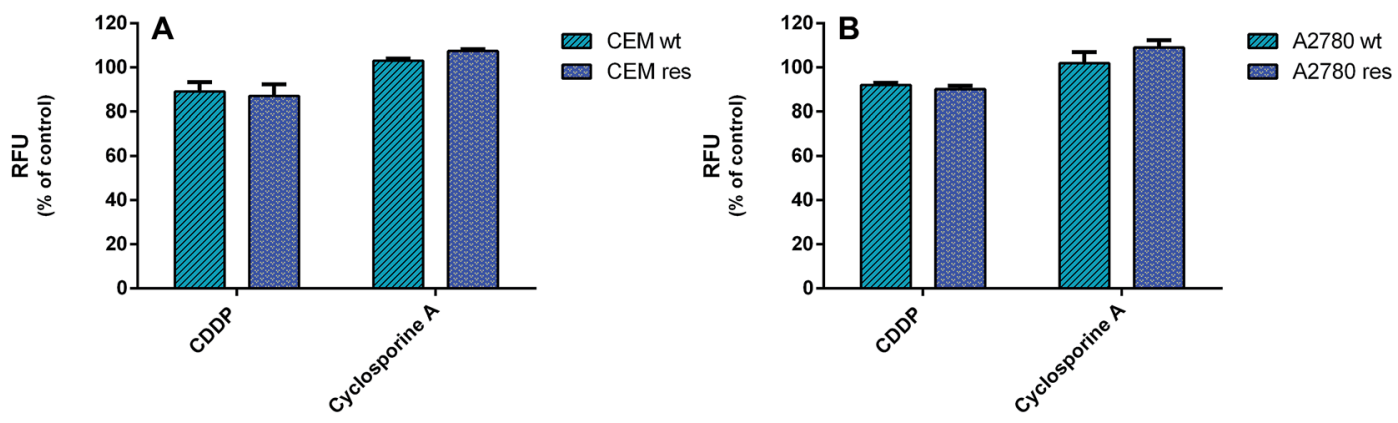

Fig. 9 Multi-drug resistance (MDR) activity. The activity of drug-efflux proteins MDR1/P-gp and MRP1 in wild type and CDDP-resistant ccrf-cem and A2780 cells was determined by a fluorimetric MDR assay kit (Abcam). Intracellular fluorescence was determined in $2 \times 10^{6}$ cells per well after $1 \mathrm{~h}$ of incubation. Data are representative of three independent experiments and are expressed as \% of untreated cells \pm SD. (A) CCRF-CEM wt treated with $0.5 \mu \mathrm{m}$ CDDP and CCRF-CEM res treated with CDDP $2.5 \mu \mathrm{m}$. (B) A2780 wt treated with CDDP $0.5 \mu \mathrm{m}$ and A2780 res treated with CDDP $2 \mu \mathrm{m}$. 

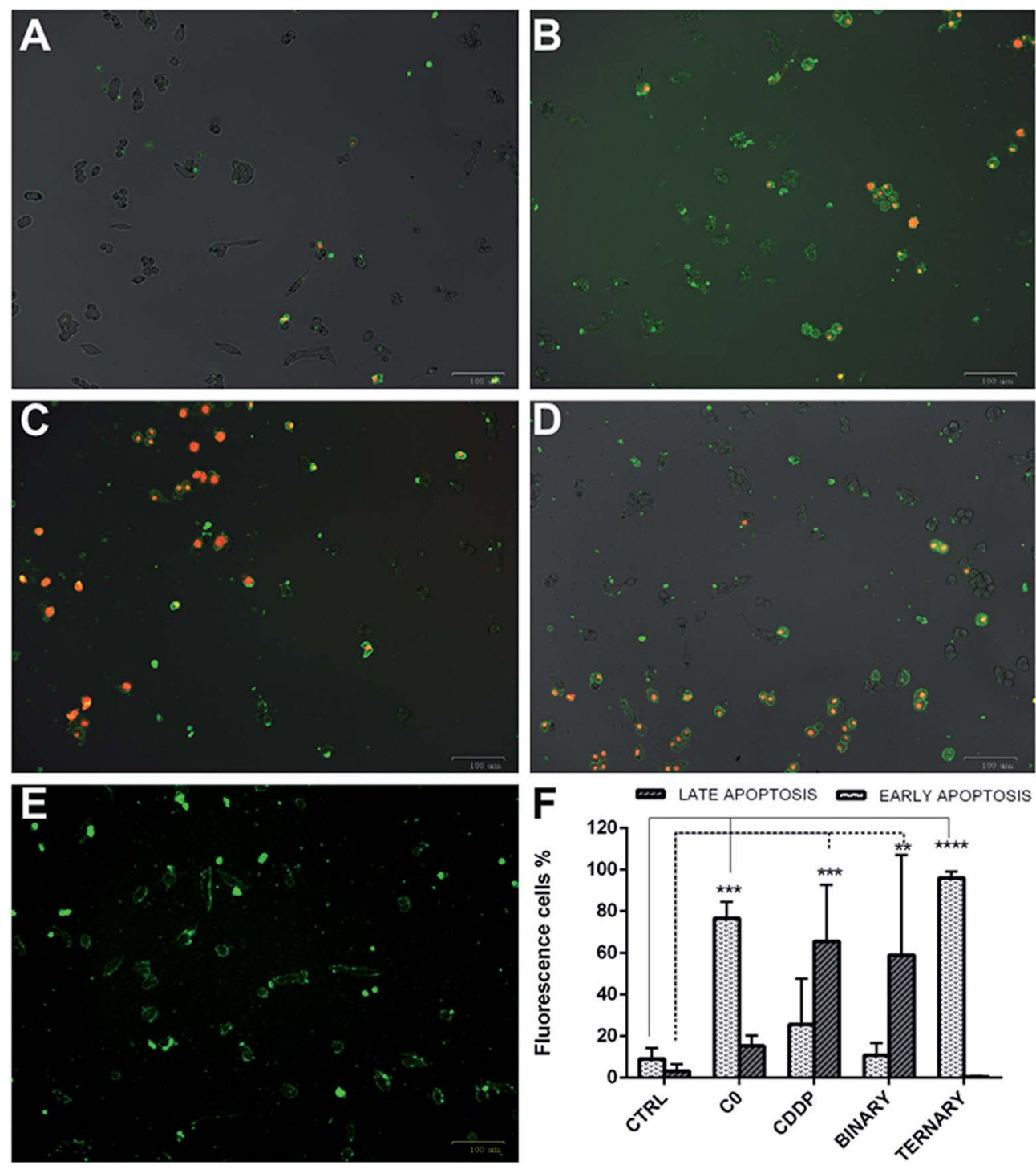

Fig. 10 Early and late apoptotic A2780-res cells treated with: C0 and CDDP alone, in dual combination, and in ternary combination with GSH. (A) Untreated controls; (B) cells treated with C0; (C) cells treated with CDDP; (D) cells co-treated with CDDP and C0; and (E) cells treated with the ternary drug combination; (F) percentages of apoptotic A2780-res cells treated with C0, CDDP and GSH, alone, in binary (CDDP + C0), and ternary $(\mathrm{CDDP}+\mathrm{CO}+\mathrm{GSH})$ combinations. Values represent the mean $\pm \mathrm{SD}$ of three independent experiments. Statistical analysis was performed by anova; ${ }^{*} p<0.05 ; * *<0.001 ; * * *<0.0001$. (A-E) Cells were imaged with the ZOE Fluorescent Cell Imager after 20 hours of incubation (A) without drugs, or with (B) C0, $0.2 \mu \mathrm{m}$; (C) CDDP, $4.0 \mu \mathrm{m}$; (D) C0 and CDDP, $0.2 \mu \mathrm{m}$ and $4.0 \mu \mathrm{m}$; (E) C0 and CDDP and GSH, $0.2 \mu \mathrm{m} ; 4.0 \mu \mathrm{m} ; 300$ $\mu \mathrm{m}$. Figures $(A-E)$ are merged images of the brightfield, red and green fluorescence channels. Early apoptotic cells were stained by Alexa Fluor 488 Annexin $V$ (green), late apoptotic cells were stained by Annexin $V$ and pi (green-red).

Table 3 Number and type of drug cocktails prepared

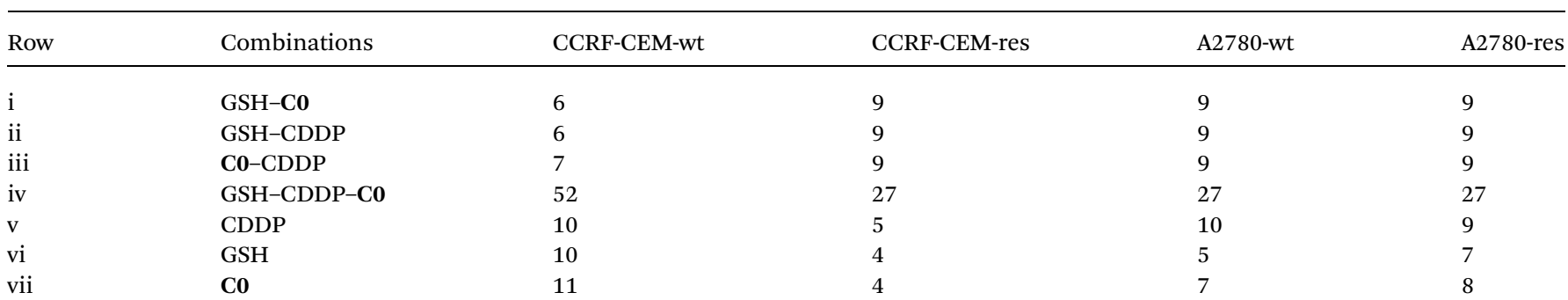


result from the ability of $\mathbf{C O}$ to target, as has been reported for other copper-phen complexes, ${ }^{\mathbf{4 0 , 4 1}}$ hexokinase (HK), a key enzyme of glycolysis. The Co-induced HK inhibition, combined with the anti-PDK activity of CDDP, may reinforce the flux of pyruvate into the Krebs' cycle that, in turn, leads to increased ROS generation, including in CDDP-resistant cells overexpressing HK. At present, we are not able to identify the main and/or specific target(s) of Co underlying its toxic mode of action in both wild type and CDDP-res cells, nor that determining its ability to overcome the antagonistic effect of GSH on CDDP. As CDDP, in fact, also $\mathbf{C o}$ has showed to act at various molecular levels, e.g. beside DNA intercalation, redox reactions with GSH (endogenous or exogenous), which also lead to the release of free and active phen molecules ${ }^{\mathbf{2 6 , 4 2}}$ and the likely activation of factor(s) of the apoptotic cascade (this work). Additionally, the copper complexes with two phen molecules have a redox potential higher than the corresponding complexes with only one phen. ${ }^{43}$

The ability of $\mathbf{C o}$ to overcome the antagonistic effect of GSH on the cytotoxic activity of CDDP fits also with the relative stability of the complexes, previously studied in solutions containing Co, CDDP and GSH, in both binary and ternary combinations..$^{38,42} \mathrm{CDDP}$ was observed to react with the copper complex, as well as with GSH or oxidized glutathione (GSSG); however, in mixtures containing simultaneously CDDP, GSH and $\mathbf{C o}$ or CDDP, GSSG and Co, only the Cu(phen)-GSH or $\mathrm{Cu}$ (phen)-GSSG complexes were detected while no platinumglutathione adducts were found. In summary, although further and finalized experiments to dissect each drug-drug interaction have to be done, the key drug for all the above effects appears to be the copper-phen complex $\mathbf{C 0}$, and its interactions with GSH and/or with factors of molecular pathways directly or indirectly involving GSH. As a matter of fact, in the presence of exogenous GSH, the cytotoxicity increase was observed only in cells simultaneously exposed to $\mathbf{C o}$.

\section{Conclusions}

The involvement of GSH in the cellular resistance to the CDDP cytotoxic effect was confirmed by our experiments in both leukaemia and ovarian carcinoma derived cell lines. In our cell models, the antagonistic effect of GSH on the cytocide activity of CDDP was particularly evident at GSH concentrations $\geq 500$ $\mu \mathrm{M}$. The simultaneous presence of the copper complex $\mathbf{C 0}$ restored the sensitivity of the cells to CDDP, and, at selected concentrations, led to strong synergistic effects. These findings were observed also in CDDP-resistant cell populations.

Thanks to the potential of our approach we are able to state that: (i) GSH and CDDP interact mainly antagonistically; (ii) Co and CDDP interact synergistically; (iii) Co and GSH interact synergistically; (iv) in the presence of $\mathbf{C o}$, at concentrations ranging from $0.10 \mu \mathrm{M}$ to $0.20 \mu \mathrm{M}$, the antagonistic effect of $\mathrm{GSH}$ on CDDP was overcome, and its cytotoxicity fully restored; and (v) the Co-CDDP-GSH system showed synergistic toxic effects, even in the presence of $1000 \mu \mathrm{M} \mathrm{GSH}$ and $0.25 \mu \mathrm{M} \leq \mathbf{C 0} \leq 0.60$ $\mu \mathrm{M}$.
These outcomes may open a chance to reach maximum therapeutic effects, reduce the frequency of drug-resistant cell mutants and, using lower doses of cisplatin, minimize side effects, by choosing the optimal drug-combination and/or the dose of $\mathbf{C 0}$ required to revert GSH-antagonism (according to the modification of the intracellular content of GSH during cycles of chemotherapy of each individual patient, personalized therapy). The synergistic cytocide activity of the three-drug cocktail in the ovarian carcinoma A2780 cell line, especially against the CDDPresistant subline and the higher cytotoxic potency exerted against leukemic cells with respect to normal lymphocytes are very promising findings. The effect of GSH in binary combinations of CDDP and C0 against two different types of CDDPresistant cell populations was confirmed and a close in vitro estimation of the overall toxic effect of the three-drug cocktail for bone marrow cells in various stages of activation was obtained. Interestingly, compared to actively proliferating normal lymphocytes (PHA/IL2-PBLs), leukaemia cells were in fact extremely more susceptible to the cytocide effect of the threedrug combination and underwent the dying process(es) much quicker.

When the cells were exposed to dual-drug combinations with CDDP and Co, mainly late apoptotic effects were observed, similar to the effect of CDDP single-drug treatments, suggesting that DNA interactions with Co-cisplatin complexes, and/or metabolism-generated ROS, trigger a process of programmed cell death. On the other hand, the ternary combination induced apoptotic effects similar to that shown by $\mathbf{C O}$ in single drug treatments. One possible explanation is that Co and CDDP compete for GSH-binding before entering the cells. This could explain why most cells showed early apoptosis. Alternatively, GSH might bind with higher affinity Co than CDDP, leaving more CDDP free molecules. All things considered, although Co, alone and in combination, induced apoptosis in both early and late phases, GSH in combination with Co and CDDP clearly triggers a statistically significant apoptotic process(es) through a pathway which do not compromise the integrity of the plasma membrane of cells. In conclusion, studies attempting to unveil the mechanism of the potentiating effect of GSH on the synergistic combination CDDP-CO and to investigate in mouse models, overall toxicity and antitumor efficacy of the multidrug cocktails, are a possible perspective of the findings reported in this manuscript.

\section{Experimental section}

\section{Materials}

Acetonitrile, cisplatin (CDDP), copper(II) basic carbonate $\left(\mathrm{Cu}_{2}\left(\mathrm{CO}_{3}\right)(\mathrm{OH})_{2}\right)$, dimethyl sulphoxide (DMSO), doxorubicin (doxo), ethanol, ethyl ether, glutathione (GSH), perchloric acid, 1,10-phenanthroline monohydrate, trypan blue, staurosporine and Interleukin 2 were purchased from Sigma-Aldrich (Italy). Foetal bovine serum (FBS), phytohemagglutinin (PHA) and kanamycin sulphate were purchased by Gibco-Invitrogen (Milan, Italy). RPMI 1640 with stable L-glutamine was purchased by EuroClone (Italy). All reagents were used without any further purification. Stock solutions of $\mathbf{C 0}$ and 
Staurosporine were prepared in DMSO, at $1000 \times$ the highest concentration to be used on the cell culture and stored at $4{ }^{\circ} \mathrm{C}$ in the dark. CDDP stock solution, being stable only for a few hours and showing a decrease in the cytotoxic potency during that time, was prepared fresh immediately before experiments took places. GSH stock solution was prepared in RPMI medium, filtered and used over a few days (the stability of the solution was checked by UV absorbance). The compound Co was prepared as previously reported. ${ }^{26}$

\section{Cell lines}

The human acute T-lymphoblastic leukaemia cell line (CCRFCEM-wt) was purchased from the American Type Culture Collection (ATCC, USA) whereas the CCRF-CEM subline resistant to $5 \mu \mathrm{M}$ CDDP (CCRF-CEM-res) was selected by us by serial sub-cultivation of the parental wild type cells at increasing concentrations of CDDP. CCRF-CEM cells were maintained in culture between $1 \times 10^{5}$ cells per $\mathrm{mL}$ and $2 \times 10^{6}$ cells per $\mathrm{mL}$, in RPMI medium with stable L-glutamine medium supplemented with $10 \%$ fetal bovine serum and $1 \%$ kanamycin (growth medium). The human ovarian carcinoma cell lines, A2780-wt and A2780-res (CDDP-resistant), were a generous gift by Dr Eva Fischer (Tumor Biology Laboratory, The Ion Chiricuta Oncology Institute, Cluj-Napoca, Romania, acquired from the European Collection of Authenticated Cell Cultures (ECACC) through Sigma Aldrich, St. Louis, USA) and were maintained in culture between $1.5 \times 10^{5}$ cells per $\mathrm{mL}$ and $3 \times 10^{6}$ cells per $\mathrm{mL}$ ( $\sim 70 \%$ confluency) in the same medium. In addition, CDDPresistant cell lines, CCRF-CEM-res and A2780-res, were treated with CDDP at $5 \mu \mathrm{M}$ (every passage) and $1 \mu \mathrm{M}$ (every two or three splits), respectively, in order to maintain the drug resistance. Cisplatin resistance was checked every month by determining the $\mathrm{CC}_{50}$ of CDDP in the absence and presence of the drug. All experiments with CDDP-resistant sublines were performed using cell populations that were grown for one passage without the drug. All cells used in the experiments were replaced every three months with freshly thawed cells from aliquots stored in liquid nitrogen. The absence of mycoplasma contamination was periodically monitored. ${ }^{44}$

\section{Cisplatin-resistant CCRF-CEM subline}

A CCRF-CEM cell line able to grow to the same extent in the absence and presence of $5 \mu \mathrm{M}$ CDDP (CCRF-CEM-res) was obtained by serial passages of parental (wild-type) cells in the presence of increasing CDDP concentrations, starting from a sub-inhibitory concentration $(0.5 \mu \mathrm{M})$. At each cell passage (every 3-4 days), the number of viable cells in cisplatin-treated cultures was compared to that of untreated cultures cultivated in parallel. Initially, and up to $1.50 \mu \mathrm{M}$, the CDDP concentration was increased by $0.25 \mu \mathrm{M}$ at each cell passage. From then on, cisplatin-treated cultures grew poorly and much more slowly than their untreated counterparts; thus, before further increasing the drug concentration, cells had to be grown at the same CDDP concentration until the cell population had regained its original growth timing and viability (about 5 consecutive passages). Given that cells never survived at concentrations higher than $5 \mu \mathrm{M}$, the cell population was stabilised by 15 further passages at this concentration, then amplified, grown for one passage in the absence of the CDDP, and stored in aliquots in liquid nitrogen for experimental use.

The number of viable cells was determined at each cell passage by the trypan blue exclusion method. At intervals during the selection process, the level of CDDP resistance was checked by the 3-(4,5-dimethylthiazol-2-yl)-2,5-diphenyltetrazolium bromide (MTT) method in cells that had grown without the drug for one passage. Doxorubicin was used as a reference compound to evaluate the cisplatin-resistance specificity. The Resistance Index (RI) was the ratio of the $\mathrm{CDDP} \mathrm{CC}_{50}$ in CCRF-CEM-res and CCRF-CEM-wt.

\section{Determination of intracellular glutathione}

Basal intracellular GSH levels were determined by using the GSH/GSSG ratio detection assay kit (Fluorometric green; Abcam ab138881, Cambridge, UK) according to the manufacturer's protocol. Briefly, CCRF-CEM wt, CCRF-CEM res, A2780 wt, and A2780 res cells were seeded at a density of $4 \times 10^{5}$ cells per $\mathrm{mL}$ in flat-bottomed 24-well plates in growth medium and allowed to settle overnight before lysis with PBS/0.5\% NP-40 (Sigma Aldrich). Cell lysates were deproteinized with the TCA/ $/ \mathrm{NaHCO}_{3}$ method and diluted to ensure readings within the standard curve values. The samples were transferred into 96-well plates with black walls and clear bottom. GSH detection was at Ex/Em $=490 / 520 \mathrm{~nm}$ by a plate reader (FLUOstar Omega, BMGLabtech). All determinations were performed in triplicate.

\section{Peripheral blood lymphocytes}

Peripheral blood lymphocytes (PBLs) used in the study were isolated from blood samples voluntarily donated by three coauthors following their informed written consent to the use for research purpose. PBLs were isolated by gradient separation in Lympholyte-H (Cedarlane). After extensive washing, cells were suspended $\left(1 \times 10^{6}\right.$ cells per $\left.\mathrm{mL}\right)$ in RPMI-1640 10\% FBS and incubated overnight. For evaluations in resting PBLs, $1 \times$ $10^{5}$ cells per well of flat-bottomed 24 -well plates were incubated in RPMI-1640 with 10\% FBS, in the absence or presence of the test drugs at the indicated concentrations.

For experiments with activated and proliferating PBLs, $1 \times$ $10^{5}$ cells per well of flat-bottomed 24 -well plates were incubated in RPMI-1640 10\% FBS supplemented with PHA $\left(2.5 \mu \mathrm{g} \mathrm{mL}^{-1}\right)$, or with PHA $\left(2.5 \mu \mathrm{g} \mathrm{mL}^{-1}\right)$ and Interleukin 2 (IL2, $\left.5 \mathrm{U} \mathrm{mL}^{-1}\right)$, in the absence or presence of the compounds at the indicated concentrations. Cell growth was determined after $24 \mathrm{~h}, 48 \mathrm{~h}$ and $72 \mathrm{~h}$ of incubation at $37{ }^{\circ} \mathrm{C}$ and $5 \% \mathrm{CO}_{2}$ through the viable cell counting with the trypan blue exclusion method. Values in drug-treated samples were expressed as percentages of their respective controls.

\section{Cytotoxic assays}

The biological stability of the copper complex (C0) solutions was checked verifying the cytotoxic activity measured by using the same solutions over more than 6 months. The tested compound maintained the same $\mathrm{CC}_{50}$ (concentration of compound that 
reduces the viable cell by $50 \%$ with respect to untreated cells) in all the performed experiments. Dilutions of the drug stocks for biologic investigations were made in RPMI medium at $2 \times$ the final concentration for single drug evaluations, or at $4 \times$ the final concentration for evaluation of binary and ternary drug combinations. The concentration of DMSO in the cells was never higher than $0.1 \%$. The effects of the drugs and drug combinations were evaluated in cultures of exponentially growing cells; for experiments in cisplatin-resistant cell cultures, cells were allowed to grow in the absence of the drug for one passage. CCRF-CEM-wt and CCRF-CEM-res cells were seeded at a density of $1 \times 10^{5}$ cells per well in flat-bottomed 24 well plates in growth medium, and simultaneously exposed to the drugs, or drug combinations. A2780-wt and A2780-res cells were seeded at a density of $1 \times 10^{5}$ cells per well in flatbottomed 24-well plates and allowed to adhere overnight before of the addition of the drug combinations. Cell growth in the absence and presence of drugs was determined in both cell lines after $96 \mathrm{~h}$ of incubation at $37^{\circ} \mathrm{C}$ and $5 \% \mathrm{CO}_{2}$, through the viable cell counting with the trypan blue exclusion method. ${ }^{45}$ This method was used because coloured GSH solution interfered with the MTT method. Values obtained in drug-treated samples were expressed as percentages of those of their respective controls. All experiments were repeated three times. Dose-response curves for each drug were determined and the $\mathrm{CC}_{50}$ of single drug and drug combinations were calculated. To evaluate the cytotoxic effects of CDDP in combination with Co and GSH, the ED-ANNs method was used, as described below.

\section{Experimental design}

The number of prepared drug cocktails are reported in Table 3 . Solutions (i), (vi) and (vii) were used to study by ANN the GSHCo system; solutions (ii), (v) and (vi) were used to study the GSH-CDDP system; solutions (iii), (v) and (vii) were used to study the CDDP-Co system; solutions (iv), (v), (vi) and (vii) were used to study the ternary CDDP-GSH-Co system. The experimental designs (ED) used are shown in the ESI (Fig. S2). $\dagger$

\section{Multi-drug resistance (MDR) activity}

The activity of major drug-efflux proteins MDR1/P-gp and MRP1 was determined by a fluorimetric MDR Assay kit (ab112142, Abcam, Cambridge, UK) according to the manufacturer's instructions. CCRF-CEM wt, CCRF-CEM res, A2780 wt and A2780 res cells were seeded at a cell density of $2 \times 10^{5} /$ well into 96-well plates, with black walls and clear bottoms, and incubated overnight. Cells were then treated with different concentrations of CDDP and Cyclosporine A (control) and the MDR dye-loading solution was added. After 1 hour incubation at room temperature in the dark, the intracellular-trapped fluorescence was detected using a plate reader (FLUOstar Omega, BMGLABTECH) with a filter set of Ex/Em $=490 / 525 \mathrm{~nm}$. The experiments were performed in triplicate.

\section{Apoptosis}

A number of $2.5 \times 10^{4} \mathrm{~A} 2780$ cells were seeded in flat-bottomed 24/well plates in $1 \mathrm{~mL}$ of supplemented growth medium as described in the cell lines section. Cells were allowed to adhere overnight before the addition of drugs or drug combinations. After 20 hours of incubation with and without the drugs, apoptosis was evaluated by Alexa Fluor 488 Annexin V and propidium iodide (PI) staining (Invitrogen), according to the manufacturer's instructions. In brief, the cells were washed twice with PBS before the addition of the binding buffer containing recombinant Annexin V conjugated to Alexa Fluor 488 dye and PI. For the quantitation of cells in early and late apoptosis, the percentage of apoptotic target cells scored with the ZOE Fluorescent Cell Imager using filters for fluorescein (FITC) and Texas Red (excitation at 480/17 nm and 556/20 nm, respectively) was calculated.

\section{Ethical statement}

All experiments were performed in compliance with institutional guidelines by the University of Cagliari. The use of human blood samples was approved for the purpose of this study by the Ethical Committee of Azienda OspedalieroUniversitaria of Cagliari, and handling of samples proceeded accordingly. Written informed consent was obtained for each individual included in the study, and all data were handled in accordance with the local ethical committee approved protocol and in compliance with the Helsinki declaration.

\section{Conflicts of interest}

There are no conflicts of interest to declare.

\section{Acknowledgements}

We wish to thank SV, DP, and AS for voluntarily donating their blood samples.

\section{References}

1 A.-M. Florea and D. Büsselberg, Cancers, 2011, 3, 1351-1371.

2 R. A. Alderden, M. D. Hall and T. W. Hambley, J. Chem. Educ., 2006, 83, 728-734.

3 N. J. Wheate, S. Walker, G. E. Craig and R. Oun, Dalton Trans., 2010, 39, 8113-8127.

4 D. Wang and S. J. Lippard, Nat. Rev. Drug Discovery, 2005, 4, 307-320.

5 C. Madeddu and A. Maccio, Expert Opin. Pharmacother., 2013, 14, 13.

6 Z. Han, J. Feng, Z. Hong, L. Chen, W. Li, S. Liao, X. Wang, T. Ji, S. Wang, D. Ma, G. Chen and Q. Gao, Biochem. Biophys. Res. Commun., 2013, 435, 188-194.

7 A. I. Ivanov, J. Christodoulou, J. A. Parkinson, K. J. Barnham, A. Tucker, J. Woodrow and P. J. Sadler, J. Biol. Chem., 1998, 273, 14721-14730.

8 F. Kratz, Metal Complexes in Cancer Chemotherapy, Germany VCH, Weinheim, 1993.

9 J. Arpalahti, in Cisplatin, Verlag Helvetica Chimica Acta, 1999, pp. 207-221. 
10 F. Legendre and J.-C. Chottard, in Cisplatin, Verlag Helvetica Chimica Acta, 1999, pp. 223-245.

11 Z. Guo and P. J. Sadler, Adv. Inorg. Chem., 2000, 49, 183-306.

12 R. B. Martin, in Cisplatin, Verlag Helvetica Chimica Acta, 1999, pp. 181-205.

13 T. W. Hambley, J. Chem. Soc., Dalton Trans., 2001, 27112718.

14 M. A. Fuertes, J. Castilla, C. Alonso and J. M. Pérez, Curr. Med. Chem.: Anti-Cancer Agents, 2002, 2, 539-551.

15 M. M. Gottesman, Annu. Rev. Med., 2002, 53, 615-627.

16 E. R. Jamieson and S. J. Lippard, Chem. Rev., 1999, 99, 24672498.

17 T. Egawa-takata, H. Endo, M. Fujita, Y. Ueda, T. Miyatake, H. Okuyama, K. Yoshino, S. Kamiura, T. Enomoto, T. Kimura and M. Inoue, Cancer Sci., 2010, 101, 2171-2178.

18 T. Ishikawa and F. Ali-Osman, J. Biol. Chem., 1993, 268, 20116-20125.

19 Y. Kasherman, S. Sturup and D. Gibson, J. Med. Chem., 2009, 52, 4319-4328.

20 A. Meister, J. Biol. Chem., 1988, 263, 17205-17208.

21 T. E. Tipple and L. K. Rogers, in Methods Mol Biol., 2012, vol. 889, pp. 315-324.

22 M. P. Gamcsik, M. S. Kasibhatla, S. D. Teeter and O. M. Colvin, Biomarkers, 2012, 17, 671-691.

23 C. Friesen, Y. Kiess and K. M. Debatin, Cell Death Differ., 2004, 11, S73-S85.

24 Z. H. Siddik, Oncogene, 2003, 22, 7265-7279.

25 T. Pivetta, M. D. Cannas, F. Demartin, C. Castellano, S. Vascellari, G. Verani and F. Isaia, J. Inorg. Biochem., 2011, 105, 329-338.

26 T. Pivetta, F. Isaia, G. Verani, C. Cannas, L. Serra, C. Castellano, F. Demartin, F. Pilla, M. Manca and A. Pani, J. Inorg. Biochem., 2012, 114, 28-37.

27 T. Pivetta, F. Isaia, F. Trudu, A. Pani and M. Manca, Talanta, 2013, 115, 84-93.

28 T. Pivetta, F. Trudu, E. Valletta, F. Isaia, C. Castellano, F. Demartin, R. Tuveri, S. Vascellari and A. Pani, J. Inorg. Biochem., 2014, 141, 103-113.

29 R. A. Davey, T. J. Longhurst, M. W. Davey, L. Belov, R. M. Harvie, D. Hancox and H. Wheeler, Leuk. Res., 1995, 19, 275-282.
30 N. Mohell, J. Alfredsson, A. Fransson, M. Uustalu, S. Byström, J. Gullbo, A. Hallberg, V. J. N. Bykov, U. Björklund and K. G. Wiman, Cell Death Dis., 2015, 6, e1794.

31 N. Nikounezhad, M. Nakhjavani and F. H. Shirazi, J. Exp. Ther. Oncol., 2017, 12, 1-7.

32 B. G. M. Vandeginste, D. L. Massart, L. M. C. Buydens, S. De Jong, P. J. Lewi and J. Smeyers-Verbeke, Handbook of Chemometrics and Qualimetrics, Part A, Elsevier Science, Amsterdam, 1997.

33 B. Donnerstag, G. Ohlenschl, J. Cinatlb, M. Amrania, D. Hofmann, S. Flindt, G. Treuschc and L. Trlgera, Cancer Lett., 1996, 110, 63-70.

34 J. B. Rotstein and T. J. Slaga, Carcinogenesis, 1988, 9, 15471551.

35 G. K. Balendiran, R. Dabur and D. Fraser, Cell Biochem. Funct., 2004, 22, 343-352.

36 A. Russo, W. Degraff, N. Friedman and J. B. Mitchell, Cancer Res., 1986, 46, 2845-2848.

37 V. Oers, Apoptosis, 1994, 1415-1420.

38 T. Pivetta, V. Lallai, E. Valletta, F. Trudu, F. Isaia, D. Perra, E. Pinna and A. Pani, J. Inorg. Biochem., 2015, 15, 107-114.

39 M. López-lázaro, Anticancer Agents Med. Chem., 2008, 8, 305312.

40 C. Granchi, D. Fancelli and F. Minutolo, Bioorg. Med. Chem. Lett., 2014, 24, 4915-4925.

41 A. Marín-Hernández, J. C. Gallardo-Pérez, S. Y. LópezRamírez, J. D. García-García, J. S. Rodríguez-Zavala, L. Ruiz-Ramírez, I. Gracia-Mora, A. Zentella-Dehesa, M. Sosa-Garrocho, M. MacÍas-Silva, R. Moreno-Sánchez and S. Rodríguez-Enríquez, Arch. Toxicol., 2012, 86, 753-766.

42 E. Cadoni, E. Valletta, G. Caddeo, F. Isaia, M. G. Cabiddu, S. Vascellari and T. Pivetta, J. Inorg. Biochem., 2017, 173, 126-133.

43 T. Pivetta, F. Isaia, G. Verani, C. Cannas, L. Serra, C. Castellano, F. Demartin, F. Pilla, M. Manca and A. Pani, J. Inorg. Biochem., 2012, 114, 28-37.

44 E. Valletta, L. Kučera, L. Prokě̌, F. Amato, T. Pivetta, A. Hampl, J. Havel and P. Vaňhara, PLoS One, 2016, 11, e0147414.

45 W. Strober, Curr. Protoc. Immunol., 2001, A.3A.1-A.3A.2. 